\title{
SOME INEQUALITIES RELATED TO $p$-SCHATTEN NORM
}

\section{Fugen GaO And MEngyu Tian}

Abstract. In this paper, we investigate the known operator inequalities for the $p$-Schatten norm and obtain some refinements of these inequalities when parameters taking values in different regions. Let $A_{1}, \cdots, A_{n}, B_{1}, \cdots, B_{n} \in B_{p}(H)$ such that $\Sigma_{i, j=1}^{n} A_{i}^{*} B_{j}=0$. Then for $0<p \leqslant 2, p \geqslant$ $\lambda>0$ and $\mu \geqslant 2$,

$$
\begin{aligned}
n^{2\left(\frac{1}{p}-\frac{1}{\lambda}\right)}\left(\sum_{i, j=1}^{n}\left\|A_{i} \pm B_{j}\right\|_{p}^{\lambda}\right)^{\frac{1}{\lambda}} & \leqslant n^{\frac{2}{p}-\frac{1}{2}}\left(\sum_{i=1}^{n}\left\|A_{i}\right\|_{p}^{p}+\sum_{i=1}^{n}\left\|B_{i}\right\|_{p}^{p}\right)^{\frac{1}{p}} \\
& \leqslant 2^{\frac{1}{p}-\frac{2}{p \mu}} n^{\frac{3}{p}-\frac{2}{p \mu}-\frac{1}{2}}\left(\sum_{i=1}^{n}\left\|A_{i}\right\|_{p^{\frac{p \mu}{2}}}+\sum_{i=1}^{n}\left\|B_{i}\right\|_{p^{\frac{p \mu}{2}}}^{\frac{2}{p \mu}} .\right.
\end{aligned}
$$

For $p \geqslant 2, p \leqslant \lambda$ and $0<\mu \leqslant 2$, the inequalities are reversed. Moreover, we get some applications of our results.

Mathematics subject classification (2010): 47B20 47A63.

Keywords and phrases: $p$-Schatten norm, operator inequality, convexity, concavity, orthogonality.

\section{REFERENCES}

[1] R. Bhatia, F. Kittaneh, Clarkson inequalities with several operators, Bull. Lond. Math. Soc. 36 (6) (2004) 820-832.

[2] R. Bhatia, F. Kittaneh, Cartesian decompositions and Schatten norms, Linear Algebra Appl. 38 (1-3) (2000) 109-116.

[3] C. MC CARThY, $c_{p}$, Israel J. Math. 5 (1967) 249-271.

[4] C. Conde, M. S. Moslehian, Norm inequalities related to p-Schatten class, Linear Algebra Appl. 498 (2016) 441-449.

[5] I. Gohberg, M. Krein, Introduction to the Theory of Linear Nonselfadjoint Operators, vol. 18, American Mathematical Society, Providence, RI, 1969.

[6] O. Hirzallah, F. Kittaneh, Non-commutative Clarkson inequalities for $n$-tuples of operator, Integral Equations Operator Theory 60 (3) (2008) 369-379.

[7] O. Hirzallah, F. Kittaneh, M. S. Moslehian, Schatten p-norm inequalities related to a characterization of inner product spaces, Math. Inequal. Appl. 13 (2) (2010) 235-241.

[8] S. MiLOSEVIC, Norm inequalities for elementary operators related to contractions and operators with spectra contained in the unit disk in norm ideals, Adv. Oper. Theory 1 (1) (2016) 147-159.

[9] M. S. Moslehian, M. Tominaga, K. S. Saito, Schatten p-norm inequalities related to an extended operator parallelogram law, Linear Algebra Appl. 435 (4) (2011) 823-829. 